\title{
Dynamic Measurements of the Position, Orientation, and DNA Content of Individual Unlabeled Bacteriophages
}

Aaron M. Goldfain, ${ }^{\dagger, / /}$ Rees F. Garmann, ${ }^{\dagger, / /}$ Yan Jin, ${ }^{\ddagger}$ Yoav Lahini, ${ }^{\natural}$ and Vinothan N. Manoharan ${ }^{\dagger, \pi, *}$

${ }^{\dagger}$ Harvard John A. Paulson School of Engineering and Applied Sciences, and "Department of Physics, Harvard University, Cambridge, MA 02138, USA

${ }^{*}$ Department of Chemistry and Biochemistry, University of California, Los Angeles, CA 90095 , USA

"These authors contributed equally

*E-mail:vnm@seas.harvard.edu

\section{Supporting Information}

In Figure S1A we show line scans of the normalized holograms of the full and empty phages from Figure 5A in the main text. We show a horizontal and a vertical line scan of each hologram because the holograms are asymmetric owing to the slight tilt of the illumination beam. Additionally, we generate a hologram of the DNA in the capsid by dividing the hologram of a phage just before ejection by its hologram just after ejection. The ejecting phage we use to generate this hologram is the phage that produced the green curve in Figure 5D of the main text. This particular phage is chosen for comparison because the focal plane of the microscope during 
this ejection is most similar to the focal plane used when recording the full and empty phage holograms. Line scans of this DNA hologram are shown in Figure S1A along with those of the full and empty phages.
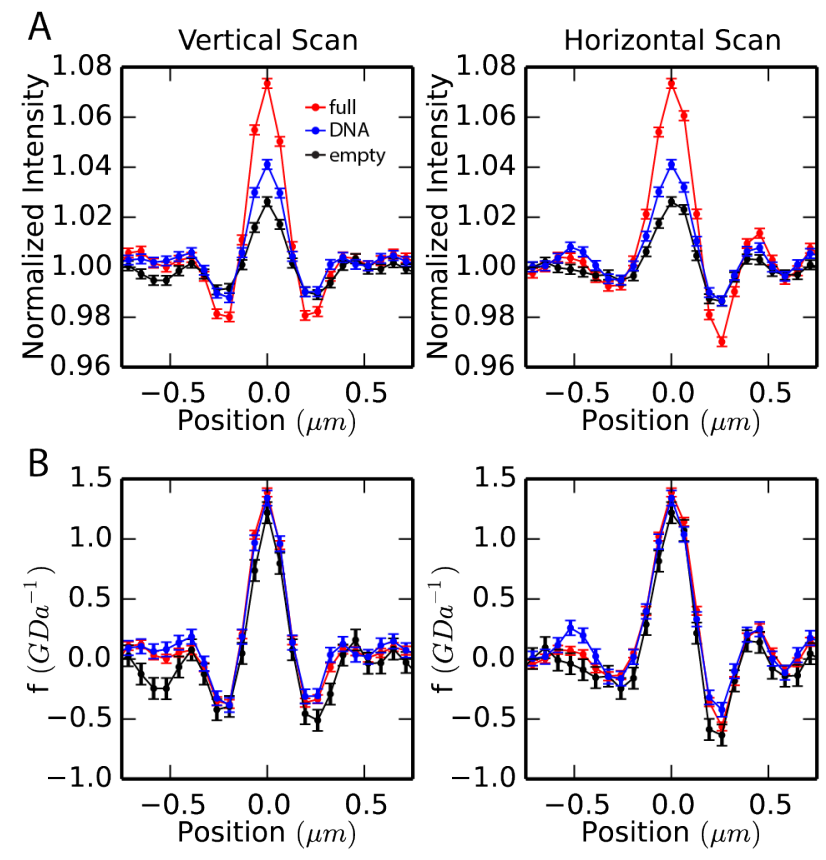

Figure S1: Vertical (left) and horizontal (right) line scans of holograms. A 10-frame time average was used for each hologram. Error bars represent the root mean square intensity of shot noise. Line scans of a full phage are plotted in red, line scans of an empty phage are plotted in black, and line scans of the DNA hologram are plotted in blue. A: Line scans of normalized holograms. B: Line scans of the hologram interference patterns scaled by the mass of the head of the phage.

In Figure S1B we show the line scans from Figure S1A scaled by the mass of each phage head. For the full and empty phages the scaled line scans were calculated by subtracting off the background and dividing by the mass of the phage head, 


$$
f_{f, e}=\frac{\left(H_{f, e}-1\right)}{m_{f, e}} .
$$

Here $f_{f, e}$ is the interference pattern from the full $(f)$ or empty $(e)$ phage normalized by the phage head mass, $H_{f, e}$ is the hologram of full or empty phage divided by an image of the reference beam, and $m_{f, e}$ is the phage head mass $\left(m_{f}=54 \mathrm{MDa}\right.$ and $\left.m_{e}=22 \mathrm{MDa}\right)$. When calculating the scaled line scans for the DNA hologram it is necessary to include the contribution of the empty phage;

$$
f_{D N A}=\frac{\left(H_{D N A}-1\right)}{m_{f}-m_{e} H_{D N A}} .
$$

Here $H_{D N A}$ is the hologram of a phage just before ejecting its DNA divided by the hologram of the same phage just after it has ejected its DNA.

The scaled line scans are quite similar to each other, particularly for the central fringes. Based on our hologram model for Rayleigh scatterers given in Equation (1) of the main text, we expect the interference patterns of different scatterers to be equivalent after scaling each by its total polarizability. The fact that the line scans of the different phage holograms overlap when scaled by the phage head mass supports our optical model of phage $\lambda$ as a Rayleigh scatterer and suggests that the total polarizability is proportional to the mass of the head of the phage. Moreover, the excellent overlap between the central fringes of all 3 scaled interference patterns supports our choice to use the normalized central fringe intensity as a measure of the DNA content of phages during ejection. The agreement of the scaled fringe pattern from the DNA 
hologram with those of the empty and full phages also indicates that ejected DNA does not significantly contribute to the hologram after ejection.

\section{Descriptions of Supporting Videos:}

S1.avi:

Holographic video of a lambda phage in TNM buffer without detergent. The phage is bound to a coverslip that was cleaned by Pyrolysis. The video is recorded at $100 \mathrm{~Hz}$ with a $1 \mathrm{~ms}$ exposure time and plays at $10 \mathrm{~Hz}$. The scale bar shows $360 \mathrm{~nm}$. The phage tail is bound securely to the coverslip but the phage head is subject to thermal motion so the hologram intensity varies from frame to frame and the hologram is blurred.

S2.avi:

Holographic video of a lambda phage in TNM buffer without detergent. The phage is bound to a coverslip functionalized with APTES. The video is recorded at $10 \mathrm{~Hz}$ with a $28 \mathrm{~ms}$ exposure time and plays at $10 \mathrm{~Hz}$. The scale bar shows $360 \mathrm{~nm}$. The red line indicates the tracked position of the phage head. The phage head translates across the coverslip due to thermal motion.

S3.avi:

Holographic video of a lambda phage in TNM buffer containing $1 \%$ oPOE detergent. The phage is bound to a coverslip functionalized with APTES. The video is recorded at $100 \mathrm{~Hz}$ with a 6.5 ms exposure time and plays at $100 \mathrm{~Hz}$. The scale bar shows $360 \mathrm{~nm}$. The red line indicates the tracked position of the phage head. The phage translates across the coverslip due to thermal 
motion. For the last 15 seconds of the video the end of the phage tail is stuck securely in place and the head is confined to the perimeter of a 180 -nm radius circle.

S4.avi:

Holographic video of a lambda phage in TNM buffer without detergent. The phage is bound to a coverslip functionalized with PEG and APTES. The video is recorded at $10 \mathrm{~Hz}$ with a $28 \mathrm{~ms}$ exposure time and plays at $10 \mathrm{~Hz}$. The scale bar shows $360 \mathrm{~nm}$. The red line indicates the tracked position of the phage head. The phage translates slightly across the coverslip due to thermal motion but is essentially fixed in place.

S5.avi:

Holographic video of a lambda phage in TNM buffer containing $1 \%$ oPOE detergent. The phage is bound to a coverslip functionalized with APTES. The video is recorded at $100 \mathrm{~Hz}$ with a 6.5 ms exposure time and plays at $100 \mathrm{~Hz}$. The scale bar shows $360 \mathrm{~nm}$. The red line indicates the tracked position of the phage head. The phage head translates across the coverslip due to thermal motion, but the end of the phage tail is stuck securely in place so the head is confined to the perimeter of a $180-\mathrm{nm}$ radius circle .

S6.avi:

Holographic video (bottom) and tracked position (top) of a lambda phage in TNM buffer containing $1 \%$ oPOE detergent. The phage is bound to a coverslip functionalized with APTES. The video is recorded at $100 \mathrm{~Hz}$ with a $6.5 \mathrm{~ms}$ exposure time and plays at $100 \mathrm{~Hz}$. The scale bar shows $360 \mathrm{~nm}$. The track is offset from the holograms so that the fringe pattern can be clearly seen when the phage head leaves the coverslip. The track is black when the phage head is on the 
surface and red when it is above the surface. The phage head translates across the coverslip due to thermal motion and stands up on its tail. 